\title{
Update on Diagnostic Difficulties in Lesions of the Minor Salivary Glands
}

\author{
Paul M. Speight
}

Received: 7 June 2007/Accepted: 19 June 2007/Published online: 30 November 2007

(c) Humana 2007

\section{Introduction}

The histopathology of the salivary glands and particularly of salivary gland tumours is complex and may be problematic for even the most experienced diagnostic pathologist [1]. The latest WHO classification [2] contains over 40 named neoplasms, many of which show significant morphological diversity resulting in overlapping features which make differentiation between tumour types difficult. Added to this, there is also a range of non-neoplastic lesions that may resemble tumours both clinically and histologically [3]. The purpose of this paper is to review some of these lesions and to highlight areas of diagnostic difficulty or controversy.

\section{Pleomorphic Adenoma}

This is the most common of the salivary gland tumours, comprising about $70 \%$ of parotid and $50 \%$ of minor gland tumours. Intra-orally, the most common site is the junction of the hard and soft palate. Because this is the most frequently encountered tumour, the features are well described and diagnosis should be straightforward. However they show considerable morphological diversity which frequently causes confusion leading to diagnostic difficulties, especially in small incisional biopsies. Also, because it is so common, it is frequently over-diagnosed leading to under diagnosis of more sinister lesions. Table 1 shows some of the features of pleomorphic adenoma. When seen

P. M. Speight $(\bowtie)$

School of Clinical Dentistry, University of Sheffield, Sheffield S10 2TA, UK

e-mail: p.speight@sheffield.ac.uk in isolation in small biopsies these features may lead to confusion with the lesion in the second column, the consequences of a misdiagnosis are clearly apparent. The pathologist must take into account the clinical history and the site of the lesion in making the diagnosis, but sometimes the characteristic morphological diversity of a pleomorphic adenoma does not become apparent until the lesion has been excised and examined in its entirety.

The key histopathological features of pleomorphic adenoma are a variable pattern of epithelium in a loosely fibrous stroma which may be myxoid, chondroid or mucoid (Fig. 1). The epithelium is usually arranged in sheets or strands, and ductal structures, often bilayered, are typical. In the minor glands, lesions are often more solid or cellular than those seen in the major glands, and the myoepithelial cells are often polygonal with a pale eosinophilic cytoplasm giving an epithelioid or plasmacytoid phenotype (Fig. 2). These cells are so typical as to be almost diagnostic and their presence in small biopsies is a helpful feature.

Pleomorphic adenomas are usually well demarcated or encapsulated, but extension of tumour into the capsule is a common feature (Fig. 3), and sometimes lobules of tumour may appear to be completely separated from the main tumour mass. This is a normal feature of this lesion and should not be misinterpreted as a sign of malignancy.

Carcinoma in pleomorphic adenoma is a well recognised entity and it is now accepted that there may be a progression to malignancy in long-standing or recurrent lesions. Overall about $6-10 \%$ of pleomorphic adenomas may progress to carcinoma. Frank carcinoma in pleomorphic adenoma is easy to diagnose and is characterised by areas of carcinoma in an otherwise typical benign pleomorphic adenoma. The malignant component is often adenocarcinoma NOS, but areas of adenoid cystic carcinoma, mucoepidermoid carcinoma or other types may be seen. In 
Table 1 Characteristic features of pleomorphic adenoma and lesions with which they may be confused

\begin{tabular}{ll}
\hline Feature & Resemblance \\
\hline Morphological diversity & $\begin{array}{c}\text { Polymorphous low grade } \\
\text { adenocarcinoma }\end{array}$ \\
$\begin{array}{l}\text { Bilayered ducts and cribriform pattern } \\
\text { Bilayered ducts with clear outer cells }\end{array}$ & $\begin{array}{l}\text { Adenoid cystic carcinoma } \\
\text { Epithelial-myoepithelial } \\
\text { carcinoma }\end{array}$ \\
Sheets of epithelioid or basaloid cells & $\begin{array}{c}\text { Basal cell adenoma or } \\
\text { adenocarcinoma }\end{array}$ \\
Myxoid stroma & $\begin{array}{l}\text { Myxoma, neural tumours } \\
\text { Chondrosarcoma }\end{array}$ \\
Chondroid stroma & $\begin{array}{l}\text { Plasmacytoma } \\
\text { Plasmacytoid cells }\end{array}$ \\
Spindled myoepithelial cells & $\begin{array}{c}\text { Sarcoma or soft tissue } \\
\text { tumour }\end{array}$ \\
Squamous metaplasia & Squamous carcinoma \\
Oncocytic metaplasia & Oncocytoma \\
\hline
\end{tabular}

From Speight and Barrett [1]

practice however the residual lesion may not be apparent or may have been obliterated by the malignant growth. Clues that a lesion is carcinoma ex pleomorphic adenoma include a long history with evidence of a previous benign lesion, areas of hyalinisation of the stroma, focal calcifications and evidence of morphological diversity in the type of carcinoma.

A key area of difficulty is in the diagnosis and significance of intra-capsular change in pleomorphic adenoma. The WHO classification recognises an entity termed noninvasive or intra-capsular carcinoma ex pleomorphic adenoma [2]. Provided the capsule has not been breached or if tumour does not extend further than $1.5 \mathrm{~mm}$ from the main tumour mass, then the lesion appears to have the same

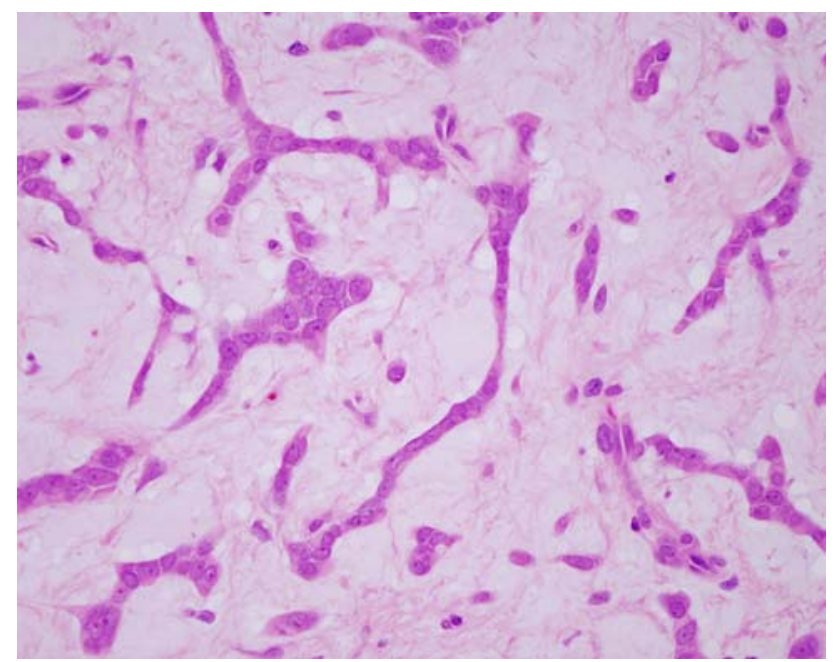

Fig. 1 Typical features of a pleomorphic adenoma include islands and strands of epithelium in a myxoid stroma

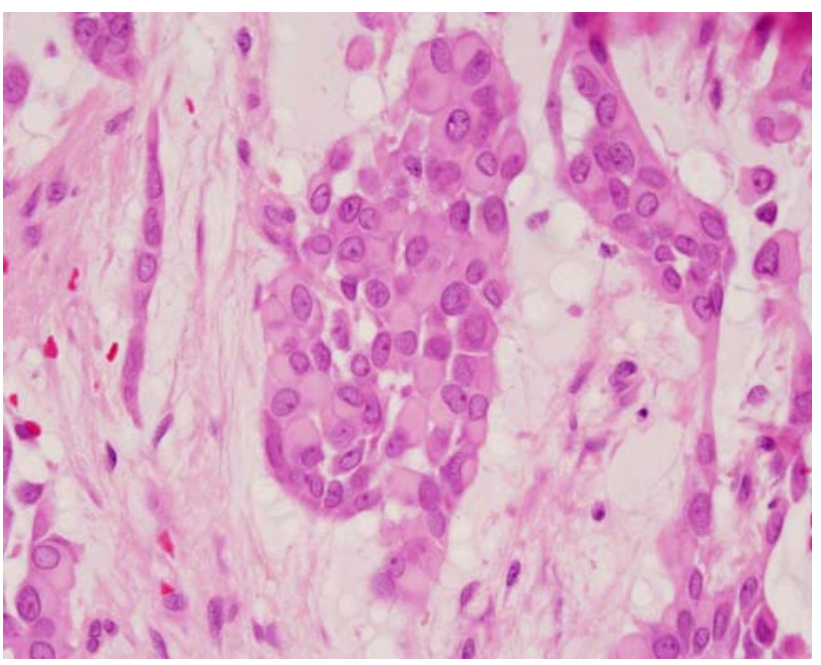

Fig. 2 Polygonal myoepithelial cells with a hyalinized cytoplasm have a plasmacytoid morphology. These cells are characteristic of pleomorphic adenoma from the palate

prognosis as a conventional benign pleomorphic adenoma. Occasional lesions show focal areas of cytological atypia, referred to as carcinoma in situ. The usual feature is of focal areas of cellular pleomorphism and nuclear hyperchromatism (Fig. 4) sometimes with increased mitoses. The atypical changes are usually within the body of the lesion and provided the lesion is excised, then prognosis is the same as for a typical benign pleomorphic adenoma. The significance of these changes is uncertain, but they provide some evidence to support the concept of a benign to malignant progression. Further evidence for this is the fact that carcinomas in pleomorphic adenomas are often aneuploid, including intra-capsular lesions [4].

\section{Polymorphous Adenocarcinoma}

This tumour is usually referred to as polymorphous lowgrade adenocarcinoma (PLGA) and is almost exclusively a tumour of minor salivary glands, with over $60 \%$ arising in the palate, and the remainder usually found in the buccal mucosa or upper lip. It was first described in 1983, but prior to that most lesions were probably diagnosed as adenoid cystic carcinoma or pleomorphic adenoma. This reflects two key features of this neoplasm-morphological diversity ('polymorphism'), and the frequent finding of cribriform or multicystic areas. Like in pleomorphic adenoma, it is the small incisional biopsy which frequently leads to misdiagnosis and on occasions even an experienced specialist pathologist cannot distinguish a small portion of a polymorphous low-grade from adenoid cystic carcinoma (Fig. 5). However, PLGA shows a characteristic 'washed out' or pale staining pattern and the nuclei are pale and open with a fine dispersed chromatin pattern. Adenoid 


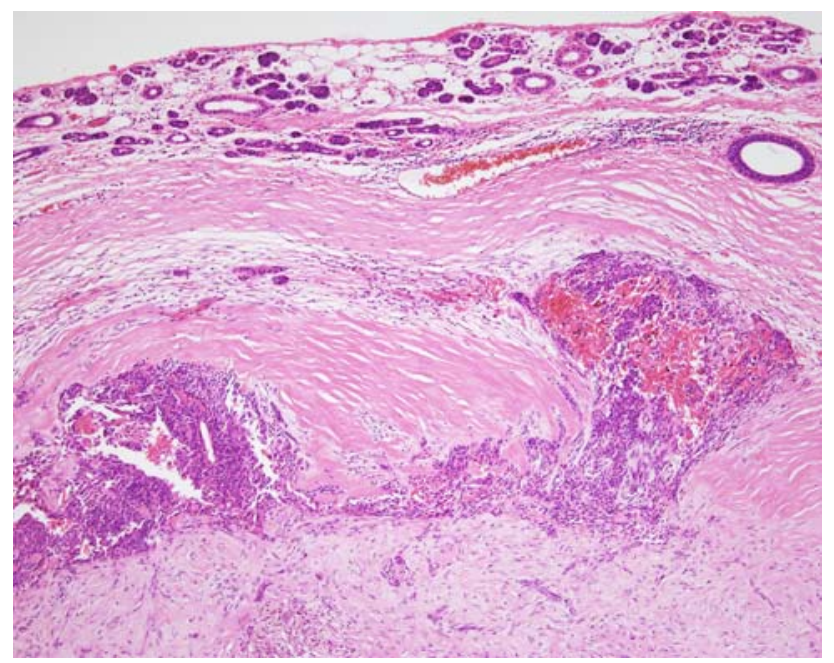

Fig. 3 Although well encapsulated, tumour protrudes into the capsule. This is typical of pleomorphic adenoma and should not be misinterpreted as malignancy

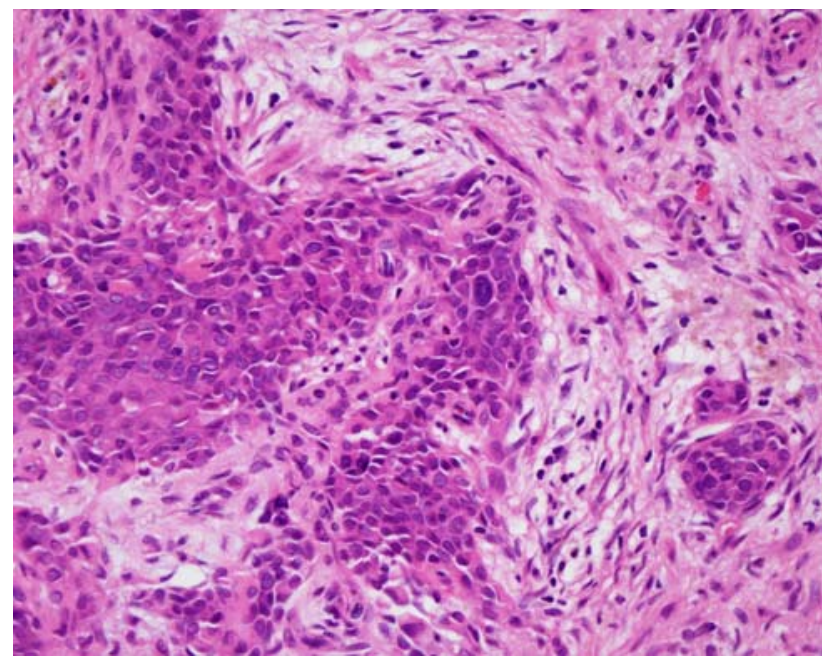

Fig. 4 Pleomorphic adenoma: a tumour island showing cytological atypia

cystic carcinoma shows small dark staining cells with hyperchromatic angular nuclei. Adenoid cystic carcinomas also show a high proliferative index and immunohistochemistry with Ki-67 uniformly shows a high number of positive cells. In our own recent studies, $30 \%$ of cells in adenoid cystic carcinoma were positive for the novel proliferation marker minichromosome maintenance protein-2 (Mcm-2). Adenoid cystic carcinomas always showed more than $10 \%$ positivity, whereas PLGA and pleomorphic adenomas were $5 \%$ and $7 \%$ positive respectively and rarely had more than $10 \%$ positive cells.

PLGA show a diverse pattern with sheets, lobules and cords of cells. Single cell filing and perineural infiltration at the periphery of the tumour is typical and a very useful diagnostic feature. Most tumours also show areas with a

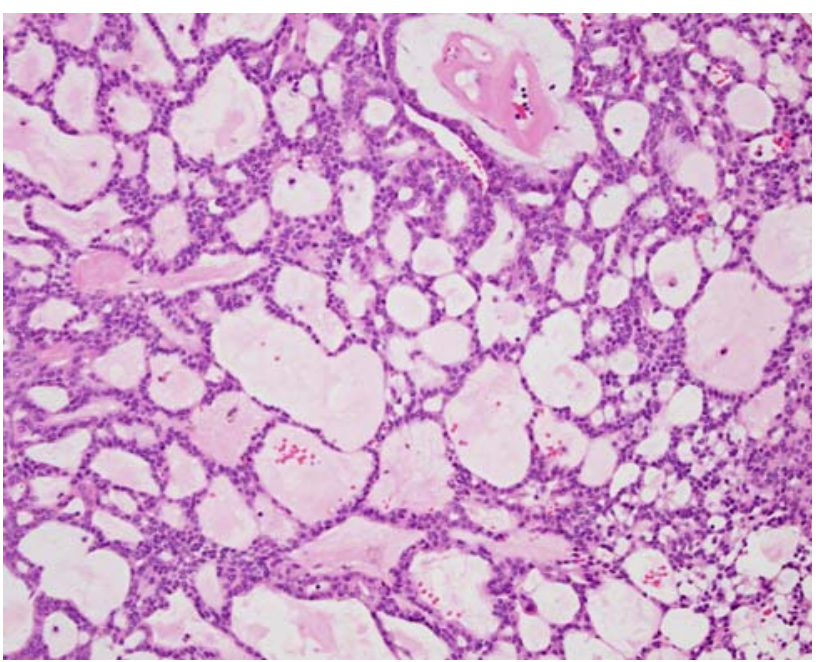

Fig. 5 A portion of a polymorphous adenocarcinoma. The multicystic cribriform pattern resembles adenoid cystic carcinoma, but the cells are pale staining with open nuclei. Many of the cystic spaces are lined by a single layer of cells

lobular pattern, often with peripheral palisading resembling basal cell lesions. Areas of cystic change are also common and a papillary cystic pattern may be encountered. Lesions which are predominantly papillary cystic are thought to have a worse prognosis and the AFIP regard these as part of the spectrum of papillary cystadenocarcinoma.

As the name suggests PLGA is regarded as a low-grade neoplasm, but in our experience, behaviour is unpredictable and similar or worse than mucoepidermoid carcinoma. Evans and Luna [5] showed that $15 \%$ of cases had cervical metastases, $7.5 \%$ had distant metastases and $12.5 \%$ of patients died of disease. An infiltrative pattern is typical and occasional cases infiltrate widely with widespread destruction at first diagnosis.

Thus, it is unclear why this lesion deserves the accolade 'low-grade' in its name and we prefer the more descriptive term polymorphous adenocarcinoma, which is not suggestive of behaviour and may help to avoid inappropriately conservative management [1]. The lesion should be managed in the same way as other salivary gland lesions, based on stage and a careful consideration of the histological features.

\section{Adenoid Cystic Carcinoma}

There are few difficulties associated with a histological diagnosis of adenoid cystic carcinoma except when small biopsies are encountered as discussed above. These tumours however may have a long and apparently indolent clinical history and pathologists and surgeons may at first be lulled into a false sense of security regarding diagnosis and management. They are aggressive widely infiltrative 
tumours with a long-term poor prognosis. The pathologist therefore needs to pay particular attention to early and accurate diagnosis. In the case of intra-oral tumours this sometimes requires determined requests for second and more adequate biopsies. For palatal lesions in particular small incisional biopsies and punch biopsies should be discouraged and all biopsies should be full thickness down to periosteum.

Histological grading of adenoid cystic carcinomas is useful and is usually straightforward because it depends on a simple analysis of the morphological pattern of the tumour [6]. The cribriform or tubular pattern predominates in about $80 \%$ of lesions and is regarded as having a better prognosis. The solid variant is regarded as high grade and recurrence rates of up to $100 \%$ have been reported and survival at 10 years is less than $10 \%$. Although low-grade tumours may do better at 5 and 10 years, grade does not predict long-term outcome which is uniformly bad with survival after 10 years of less than 50\% [7].

\section{Mucoepidermoid Carcinoma}

In most series mucoepidermoid carcinoma is the most common malignant salivary gland tumour, representing about $10 \%$ of salivary gland tumours and about $25 \%$ of malignant lesions. Although most common in the parotid gland they are frequently encountered in the oral cavity in the palate, cheeks, lips, tongue or retromolar region. An important feature of this lesion is that it is the most common salivary malignancy found in children. Since they are frequently cystic, incisional biopsies of lesions from children are occasionally misdiagnosed as mucoceles. In this respect it should be noted that mucoceles are almost always encountered on the lower lip-cystic biopsies from the palate or retromolar region should therefore be considered carefully before a diagnosis is made.

Histological diagnosis of this tumour is based on the identification of an admixture of epidermoid cells and mucous cells. The most frequent pattern is a multicystic tumour with cystic spaces lined by duct-like or epidermoid cells and mucous cells. In almost all cases more solid areas are also seen composed of sheets of epidermoid or bland intermediate cells with scattered mucous cells (Fig. 6). Clear cells are also seen and occasional lesions may be predominantly clear. The cystic pattern is typical of lowgrade lesions and is seen in over $70 \%$ of cases. Intermediate and high-grade lesions show more solid areas and an increased proportion of epidermoid cells. High-grade lesions may also show cytological atypia, perineural infiltration, necrosis and a more invasive growth pattern. Focal keratinisation and squamous carcinoma-like areas may also be seen.

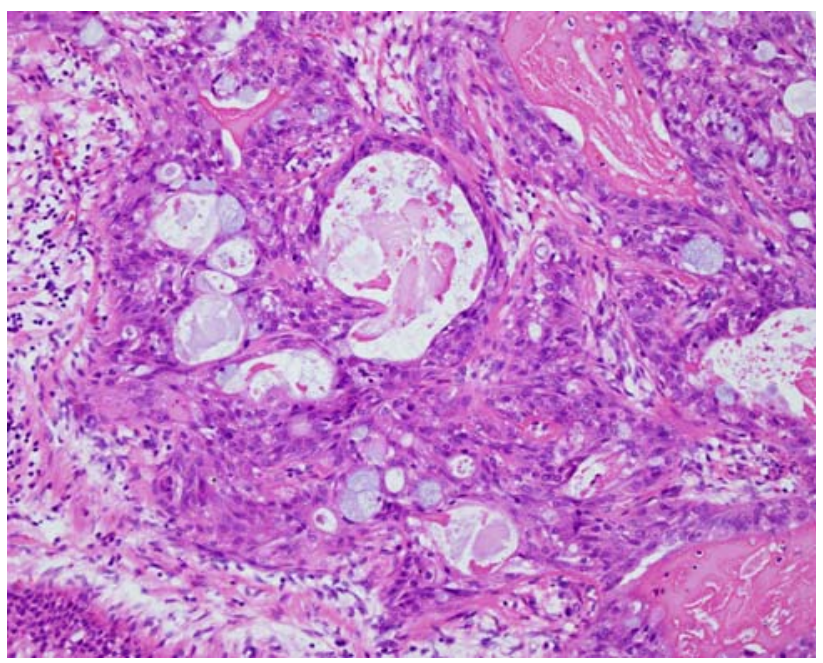

Fig. 6 Mucoepidermoid carcinoma: there are cystic areas, but in this solid focus there is a typical mixture of epidermoid, intermediate and mucous cells

Histological grading of mucoepidermoid carcinomas is a useful prognostic indicator and the most popular scheme is that of Brandwein et al. [8]. This is based on the above features and grades lesions into low, intermediate and high grade. Using this scheme the authors showed that all patients with low-grade lesions were disease free at 10 years compared to $70 \%$ with intermediate grade and $40 \%$ with high-grade lesions.

\section{Basal Cell and Canalicular Adenomas}

These are quite common benign tumours that often involve the upper lip. They are well encapsulated lesions with characteristic monomorphic histological features. There are two principle diagnostic difficulties with these lesions. The first is differentiation from malignant basal cell or basaloid lesions in small biopsies which do not include the margins. In these cases the clinical history is helpful, but if necessary a second biopsy should be sought. The second problem area is that canalicular adenomas may be multifocal [9]. Small satellite tumours may be found and in some cases multiple lesions may be found within the lip. Such multifocality should not be confused with invasion or malignancy. Each small tumour is usually discrete and shows characteristic encapsulation.

\section{Non-neoplastic Lesions}

There are a number of non-neoplastic conditions which may present clinically or histologically like a neoplasm, as well as rare entities that may not be recognised [3]. 


\section{Sclerosing Polycystic Adenosis}

This is a rare inflammatory disorder of the salivary glands that resembles fibrocystic disease of the breast. Almost all cases have been reported in the parotid gland although rare cases have been seen in the submandibular gland and intraoral minor glands (Fig. 7). The lesions are usually well demarcated areas of densely collagenous relatively acellular fibrous tissue containing multiple cystically dilated ductal structures and residual acini. Cribriform areas may be seen and some cases have shown a papillary cystic pattern. Mucous cells, sebaceous cells and squamous metaplasia have also been seen, and there is usually an associated chronic inflammatory cell infiltrate. The lesion may resemble a range of salivary tumours including adenoid cystic carcinoma, cystadenocarcinoma, mucoepidermoid carcinoma and acinic cell carcinoma. Sclerosing polycystic adenosis is characterised by dense fibrosis, a lobular pattern and an association with inflammation. Some cases however have shown evidence of cytological atypia and even areas resembling carcinoma-in-situ. No cases however have become malignant and the significance of these findings remain uncertain. Careful review and followup is however advisable.

\section{Necrotizing Sialometaplasia}

This is a well described entity that resembles malignancy both clinically and histologically. Although well known it continues to cause diagnostic confusion and misdiagnosis has obvious severe consequences. Although the cause is not known it appears to be a reactive process and infarction

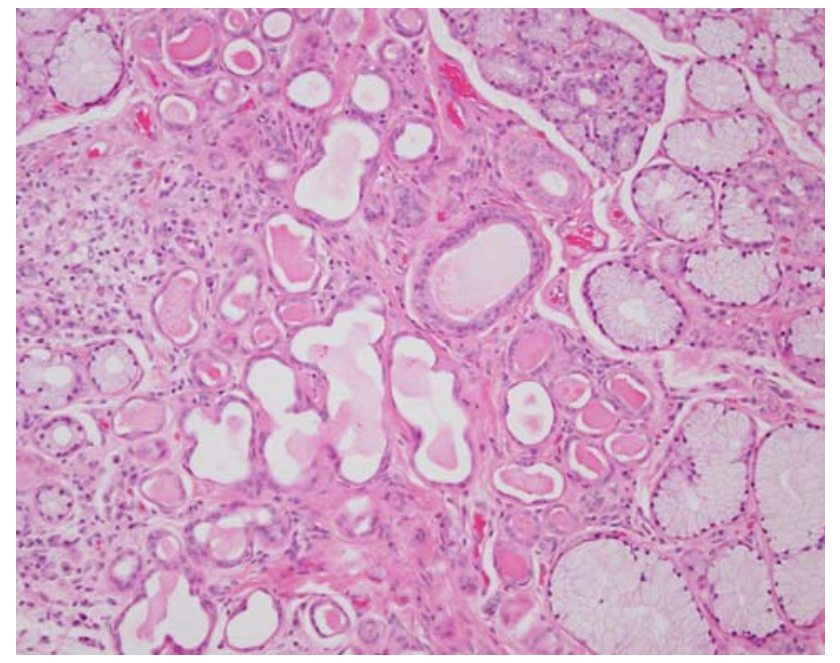

Fig. 7 A small focus of sclerosing polycystic adenosis in an inflamed minor salivary gland. Cystically dilated ducts are arranged in a dense fibrous connective tissue. Areas of inflammation and atrophy can be seen at the periphery has been suggested as the primary etiological event. Diagnostic errors are compounded by the fact that necrotising sialometaplasia may frequently be found at the surgical margins of a previous excision, making it very difficult to differentiate the lesion from a recurrence of the previous malignancy.

Necrotising sialometaplasia is most common in the minor salivary glands and the palate is the most frequent site. It presents as a firm nodule that usually ulcerates and resemble squamous carcinoma clinically. If left untreated the lesions usually heal spontaneously in 4-6 weeks. Histologically the typical appearance is of islands of squamous epithelium in an inflamed fibrous connective tissue (Fig. 8). Alone, this appearance may be very difficult to distinguish from a carcinoma, but necrotising sialometaplasia also shows areas of necrotic salivary tissue and areas of transition from normal ducts to squamous metaplasia will be evident. Cytologically the epithelium is bland and the squamous islands are typically rounded or lobular in shape with well demarcated outlines.

\section{Adenomatoid Hyperplasia}

Tumour-like swellings of the minor or major glands may be seen as a consequence of hyperplasia. In the minor glands this is most often encountered on the palate due to hyperplasia of the mucous acini. The lesion presents clinically as a sessile tumour-like swelling resembling pleomorphic adenoma. Histological diagnosis is straightforward and shows lobules of enlarged mucous acini in which the cells are filled with secretory granules and the nuclei squeezed to the basal portions. Most cases are

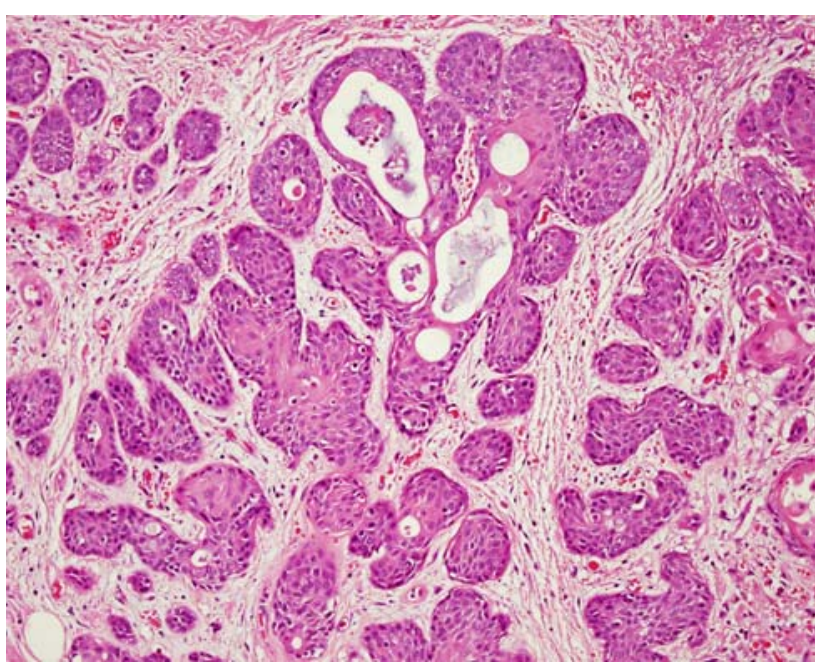

Fig. 8 Necrotising sialometaplasia: the lesion is characterised by islands of squamous epithelium. Transition from ducts to squamous metaplasia can be seen 
associated with focal inflammation and ductal dilatation and a history of trauma is often elicited [10]. There is a need for some caution however because occasional lesions are associated with an adjacent salivary gland tumour. The pathologist and clinician must therefore be confident that the sample is representative of the clinical lesion.

\section{Salivary Gland Cysts}

Mucoceles are the most common cystic lesions of the salivary glands and rarely present any diagnostic difficulties. Almost $90 \%$ of mucoceles are of the extravasation variety and are most frequently encountered in children and on the lower lip. Retention mucoceles are more rare, but are most often found in patients over 40 years and may present in the floor of the mouth, palate or buccal mucosa. The key areas for attention are that mucoceles are very rare on the upper lip, or intra-orally in children. The possibility of a cystic neoplasm should always be considered when cystic lesions from these sites present for histological analysis.

Occasionally mucous extravasation cysts may arise in the superficial lamina propria and present as a small raised blister (Fig. 9). These superficial mucoceles are often multiple and appear to be more common in women. They are often confused with a vesiculo-bullous disorder, particularly mucous membrane pemphigoid [11]. Histologically a simple mucin stain will clarify the nature of the lesion.

\section{Summary and Conclusions}

The salivary glands may present with a diverse range of lesions presenting a challenge to even the most experienced

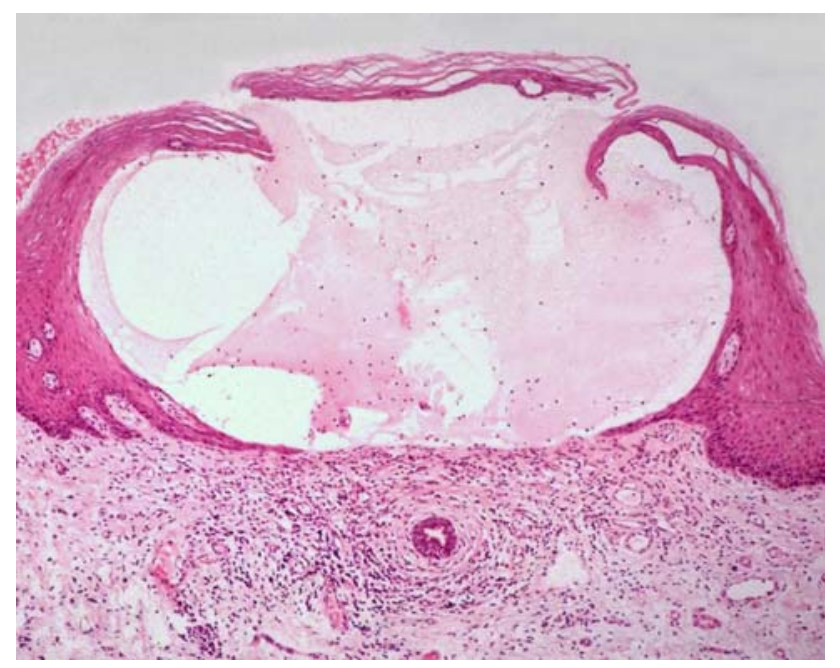

Fig. 9 A superficial mucocele. Extravasated mucin forms a subepithelial blister resembling mucous membrane pemphigoid (Courtesy of Prof John Eveson) diagnostic pathologist. Both the pleomorphic adenoma and polymorphous adenocarcinoma are characterised by morphological diversity which can result in errors if nonrepresentative samples are examined in small biopsies. Particular attention should therefore be given to the examination of an adequate tissue specimen and small biopsies and punch biopsies, especially of palatal lesions should be discouraged. The differential diagnosis of pleomorphic adenoma, adenoid cystic carcinoma and polymorphous adenocarcinoma may be particularly problematic. In general immunocytochemistry has little role to play, but the consistently high proliferation rate in adenoid cystic carcinomas can be demonstrated and may be useful in equivocal cases.

A range of non-neoplastic lesions may resemble tumours and the pathologist must give special attention to the clinical history and the age of the patient, especially in distinguishing benign cystic lesions from cystic neoplasms and determining the correct diagnosis in necrotising sialometaplasia.

\section{References}

1. Speight PM, Barrett AW. Salivary gland tumours. Oral Diseases 2002;8:229-40.

2. Barnes L, Eveson JW, Reichart P, Sidransky D. World Health Organization Classification of Tumours: pathology and genetics of head and neck tumours. IARC Press, Lyon: 2005.

3. Eveson JW, Speight PM. Non-neoplastic lesions of the salivary glands: new entities and diagnostic problems. Current Diagnostic Pathology 2006;12:22-30.

4. Vargas PA, Torres-Rendon A, Speight PM. DNA ploidy analysis in salivary gland tumours by image cytometry. J Oral Pathol Med 2007;36:371-6.

5. Evans HL, Luna MA. Polymorphous low grade adenocarcinoma. A study of 40 cases with long term follow up and an evaluation of the importance of papillary areas. Am J Surg Pathol 2000;24: 1319-28.

6. Barrett AW, Speight PM. The controversial adenoid cystic carcinoma. The implications of perineural invasion. In: McGurk M, Renehan A, editors. Controversies in the management of salivary gland disease. Oxford University Press: Oxford; 2001. 211-217.

7. Spiro RH, Huvos AG. Stage means more than grade in adenoid cystic carcinoma. Am J Surg 1992;164:623-8.

8. Brandwein MS, Ivanov K, Wallace DI, et al. Mucoepidermoid carcinoma. A clinicopathological study of 80 patients with special reference to histological grading. Am J Surg Pathol 2001;25:835-45.

9. Rousseau A, Mock D, Dover DG, Jordan RC. Multiple canalicular adenomas: a case report and review of the literature. Oral Surg Oral Med Oral Pathol Oral Radiol Endod. 1999;87: 346-350.

10. Barrett AW, Speight PM. Adenomatoid hyperplasia of oral minor salivary glands. Oral Surg Oral Med Oral Pathol Oral Radiol Endod 1995;79:482-7.

11. Eveson JW. Superficial mucoceles: pitfall in clinical and microscopic diagnosis. Oral Surg Oral Med Oral Pathol 1988;66: 318-322. 